\title{
Muscle strength and body fat percentage in children and adolescents from the Maule region, Chile
}

\author{
Marco Cossio-Bolaños, M.D. ${ }^{a}$, Rossana Gómez-Campos, M.D. ${ }^{b}$, \\ Luis Felipe Castelli Correia de Campos, M.D. ${ }^{c}$, Jose Sulla-Torres, M.D. ${ }^{d}$, \\ Camilo Urra-Albornoz, Magister ${ }^{e}$ and Vitor Pires Lopes, M.D.f
}

a. Department of Sciences of Physical Activity, Universidad Católica del Maule, Talca, Chile.

b. Department of Diversity and Inclusion in the Classroom Universidad Católica del Maule, Talca, Chile.

c. Department of Educational Sciences, Universidad del Bío-Bío, Chillán, Chile.

d. Universidad Nacional de San Agustín de Arequipa, Arequipa, Peru.

e. School of Kinesiology, School of Health, Universidad Santo Tomás, Talca, Chile.

f. Research Center in Sports Sciences, Health Sciences and Human Development (CIDESD), Sport Science Department, Polytechnic Institute of Bragança, Portugal.

E-mail address:

Rossana Gómez

Campos, M.D.:

rossaunicamp@gmail. com

\section{Funding:}

None.

Conflict of interest:

None.

Received: 10-14-2019

Accepted: 6-3-2020

\section{ABSTRACT}

Objective. To analyze the association between relative handgrip strength (RHGS) and fat mass (FM) after controlling for the potential effect of maturity status.

Methodology. Both male and female children and adolescents aged $\geq 7.5$ to $\leq 15.49$ years were studied. RHGS was assessed using a hydraulic hand dynamometer. FM percentage was established using a dual-energy X-ray absorptiometry. Age and sex differences in RHGS levels between normal weight and obese participants were studied with an analysis of covariance. A covariate was years from peak height velocity (maturity status). The association between RHGS and FM levels was analyzed using a partial correlation and controlling for age at peak height velocity.

Results. A total of 1685 students (731 girls and 954 boys) participated. Four age groups were established (7.5-9.4 years, 9.5-11.4 years, 11.513.4 years, and 13.5-15.4 years). RHGS increased with age in both males and females. FM values were high in all age groups. No differences were observed in groups 3 and 4 among girls or in group 4 among boys. Participants classified as normal weight showed a significantly higher RHGS than their obese peers. A negative association was noted between RHGS and FM. Conclusion. RHGS was shown to be negatively associated with FM after controlling for the effect of maturity status.

Key words: obesity, handgrip strength, fat tissue, growth and development.

http:/ / dx.doi.org/10.5546/ aap.2020.eng.320

To cite: Cossio-Bolaños M, Gómez-Campos R, Castelli Correia de Campos LF, Sulla-Torres J, et al. Muscle strength and body fat percentage in children and adolescents from the Maule Region, Chile. Arch Argent Pediatr 2020;118(5):320-326.

\section{INTRODUCTION}

The increase in the prevalence of overweight and obesity among children and adolescents is currently one of the main concerns in public health. The worldwide forecast for 2015 regarding overweight and obesity prevalence among children aged 5 to 7 years was $15.0 \%$ and $5.4 \%$, respectively. This accounted for a $1.9 \%$ increase in the overall excess weight prevalence from 2013 in this age group. ${ }^{1}$

In developed countries, in 2013, the prevalence of overweight was $24 \%$ and $23 \%$ among boys and girls, respectively. ${ }^{2}$ These prevalence values corresponding to developing countries ranged from $8 \%$ to $13 \%$ in both males and females. ${ }^{3}$ In Chile, the latest national health survey found a $39.8 \%$ of overweight and a $31.2 \%$ of obesity. ${ }^{4}$

In general, several studies based on obese children and adolescents demonstrated that they are less active and have a lower physical fitness than their non-obese peers. Actually, the relation among physical activity (PA), sedentary activity (e.g., watching TV), physical fitness, and obesity is not clearly established; ;,5 therefore, it is relevant to study the relation between relative handgrip strength (RHGS) and fat mass (FM) in pediatric populations.

Physical fitness is a multidimensional physical concept that encompasses different components, such as cardiorespiratory fitness, strength, and muscle strength endurance, etc. It is also considered one of the most relevant markers of health ${ }^{6}$ and a predictor of morbidity 
and mortality, in association with cardiovascular diseases and all causes of death. ${ }^{7}$ Physical fitness is partially a genetic determination, but it is also highly affected by environmental factors, such as regular PA and physical exercise and their main determining factors. ${ }^{3}$

Cardiorespiratory fitness and muscle fitness appear to have a combined cumulative effect on risk factors for cardiovascular disease in adolescents. ${ }^{6}$ For example, García-Artero et al. ${ }^{8}$ confirmed that a high aerobic capacity in males and a high muscle strength in females were associated with a lower metabolic risk and that, for a specific cardiorespiratory fitness, an increased muscle strength level was associated with a lower metabolic risk in association with cardiovascular disease.

Steene-Johannessen et al. ${ }^{9}$ verified, in children and adolescents aged 9-15 years, that muscle fitness was negatively associated with metabolic factors after controlling for age, sex, and maturity status. Muscle strength is one of the components of physical fitness, which is considered a major factor in development during childhood and youth. It is the basis of game-related activities and social interaction with other children.

One of the most common ways to measure strength is handgrip strength (HGS) using a dynamometry, and has been shown to have a positive association with different medical conditions. A cross-sectional study verified that HGS was related to liver fat in adolescents with excess body fat. ${ }^{10}$ In addition, a longitudinal study conducted in adolescents after controlling for the effect of cardiorespiratory fitness, PA, and fatfree mass established that HGS was significantly associated with metabolic risk. ${ }^{11}$

Based on this evidence, and due to the high prevalence of overweight and obesity observed in Chile, this study proposes the hypothesis of a potential negative association between HGS and FM as measured by a dual-energy X-ray absorptiometry (DXA) among Chilean children and adolescents. This information may be useful for health care providers working with pediatric populations, especially during puberty, because not all adolescents mature at the same rate and pace. Our objective was to analyze the association between HGS and FM among Chilean children and adolescents after controlling for the potential effect of somatic maturation.

\section{METHODOLOGY Participants}

A descriptive, correlational study was done in male and female students from the Maule Region (Talca, Chile) in 2016. Participants were children and adolescents aged $\geq 7.5$ to $\leq 15.49$ years who were randomly selected from 12 schools located in the urban area of the Maule Region (Chile). Those with physical limitations or who had prostheses and/or metal implants in their body were excluded.

For the assessment of the study outcome measures, each participant gave their assent and their parents, their informed consent. Permission was also requested to each school administration upon explaining the study objective. The study was approved by the Ethics Committee of Universidad Autónoma de Chile.

Assessments were done in an enclosed laboratory $\left(22-24{ }^{\circ} \mathrm{C}\right)$ of the university, Mondays through Fridays from 8:30 a.m. to 12:30 p.m. All students were taken to and from the assessments, and procedures were carried out by three providers who were duly trained on anthropometric outcome measures and DXA scanning.

\section{Handgrip strength}

HGS was measured using a JAMAR hydraulic hand dynamometer (Hydraulic Hand Dynamometer $\square$, model PC-5030 J1, Fred Sammons, Inc., Burr Ridge, IL, USA). Accuracy was $0.1 \mathrm{lbf}$ for both hands. The protocol proposed by Richards et al. was followed. ${ }^{12}$ Students were in a standard position with each hand. To control for the effects of fatigue, three attempts were performed by alternating the hands with approximately 2 minutes of rest between each attempt. The better measurement was recorded for each attempt with each hand. The measurements of both hands were added to obtain an overall HGS indicator.

\section{Body size and maturity status}

All somatic measurements were obtained by two experienced anthropometrists following the protocols described by Ross et al. ${ }^{13}$ Body weight, standing height, and sitting height were taken at the laboratory with participants wearing light clothing (T-shirt, shorts, and no shoes). Body weight was measured using a digital scale (Seca Gmbh \& Co. KG, Hamburg, Germany) with an accuracy of $0.1 \mathrm{~kg}$. Standing height was measured with a stadiometer (Seca \& Co. KG, Hamburg, Germany) with an accuracy of $0.1 \mathrm{~mm}$ and a scale of $0 \pm 2.50 \mathrm{~m}$. Sitting height was taken using a wooden bench (50-cm-high) with a measurement 
scale of $0-150 \mathrm{~cm}$ and an accuracy of $1 \mathrm{~mm}$. All outcome measures were taken twice. The technical error of measurement (TEM) was $0.8 \%$ to $2 \%$ for all measurements.

Body mass index (BMI) [weight $(\mathrm{kg}) /$ height $^{2}$ $\left(\mathrm{m}^{2}\right)$ ] was also estimated. Maturity status was estimated based on years from peak height velocity (YPHV) using the anthropometric regression equation suggested by Mirwald et al. ${ }^{14}$ This technique is widely used in Chile because it is not invasive and allows to assess maturity status in a cross-sectional manner. ${ }^{15}$

\section{Body composition}

A DXA (Lunar Prodigy, General Electric, Fairfield, CT) was done following the procedures described by Kelly et al. ${ }^{16}$ and the manufacturer's instructions. All measurements were performed by a specialized technician. Before starting the DXA, the subject had to lie on the scanning table in a supine position, with their arms and legs parallel to the table. Their ankles were held with a Velcro fastening tape to ensure they remained in the standard position. Fat percentage and FM were recorded for this test.

To ensure DXA reliability, scanning was repeated shortly after the first test (on the same day) assessing $10 \%$ of the sample every 10 subjects. Inter- and intra-evaluator TEM showed values below $1.5 \%$. Overweight and obesity were defined based on body fat values obtained with the DXA with a cutoff point of $85^{\text {th }}$ percentile for overweight and $95^{\text {th }}$ percentile for obesity for each sex and age group..$^{17,18}$

At the end of the project, the study results were reported to parents. Students who had a high risk were referred to specialized pediatricians for treatment.

\section{Statistical analysis}

Four age groups were established with a 2-year interval. Group 1: 7.5-9.4 years; group 2: 9.5-11.4 years; group 3: 11.5-13.4 years; group 4: 13.5-15.4 years. RHGS was estimated by dividing HGS over body mass. After verifying data normality, a descriptive statistical analysis was performed to establish the arithmetic mean, the standard deviation, and the age and sex range.

A one-way analysis of variance (ANOVA) was done to analyze the difference between each age group in both males and females. An analysis of covariance (ANCOVA) was done to establish the differences in RHGS in relation to age group and sex between normal weight and obese participants, considering YPHV as a covariate. A partial correlation (Pearson) was done to analyze the relation between RHGS levels and FM percentage after controlling for YPHV.

\section{RESULTS}

Figure 1 shows the study sample selection process. Table 1 describes the arithmetic mean and standard deviation for the six studied outcome measures by sex and age group. No significant differences were observed between males and females across all outcome measures and age groups. Significant differences were only noted in YPHV across all age groups, and it was worth considering that girls had an advanced YPHV compared to boys. HGS increased with age in both males and females. Only boys from age group 4 reflected a significantly higher HGS than girls.

Table 2 shows the descriptive statistics (arithmetic mean and standard deviation) for FM percentage and obesity prevalence by age and sex. FM was used as a classification criterion. ${ }^{16}$ Mean FM values were high across all age groups in both males and females. In relation to the overall high prevalence of overweight and obesity, $53.6 \%$ of participants were obese. In general, girls across all groups had higher FM values than boys.

Table 3 shows HGS relative to body mass $(\mathrm{kg})$ for normal weight and obese children and adolescents by age and sex. RHGS was higher in the older age groups; therefore, it increased with age in both males and females. However, both boys and girls in group 3 showed a reduction, except for girls in groups 3 and 4 and boys in group 4. Normal weight participants had a significantly higher RHGS than obese ones.

Zero-order and partial correlations are shown in Table 4. In both cases, the effect of YPHV was controlled between RHGS and FM percentage (for age and sex). The correlations between both outcome measures were negative. The correlations were moderate; values were low and not significant only for groups 3 and 4 in girls and group 4 in boys.

\section{DISCUSSION}

HGS appears to be an adequate indicator of health because it is associated with lower risks for cardiovascular disease, both in adults and adolescents. ${ }^{6,8,11}$ Results suggest that FM percentage and RHGS as adjusted for body weight are negatively associated, and such association is even higher among younger age groups. In fact, both 
FIGURE 1. Flow chart of the sample selection process

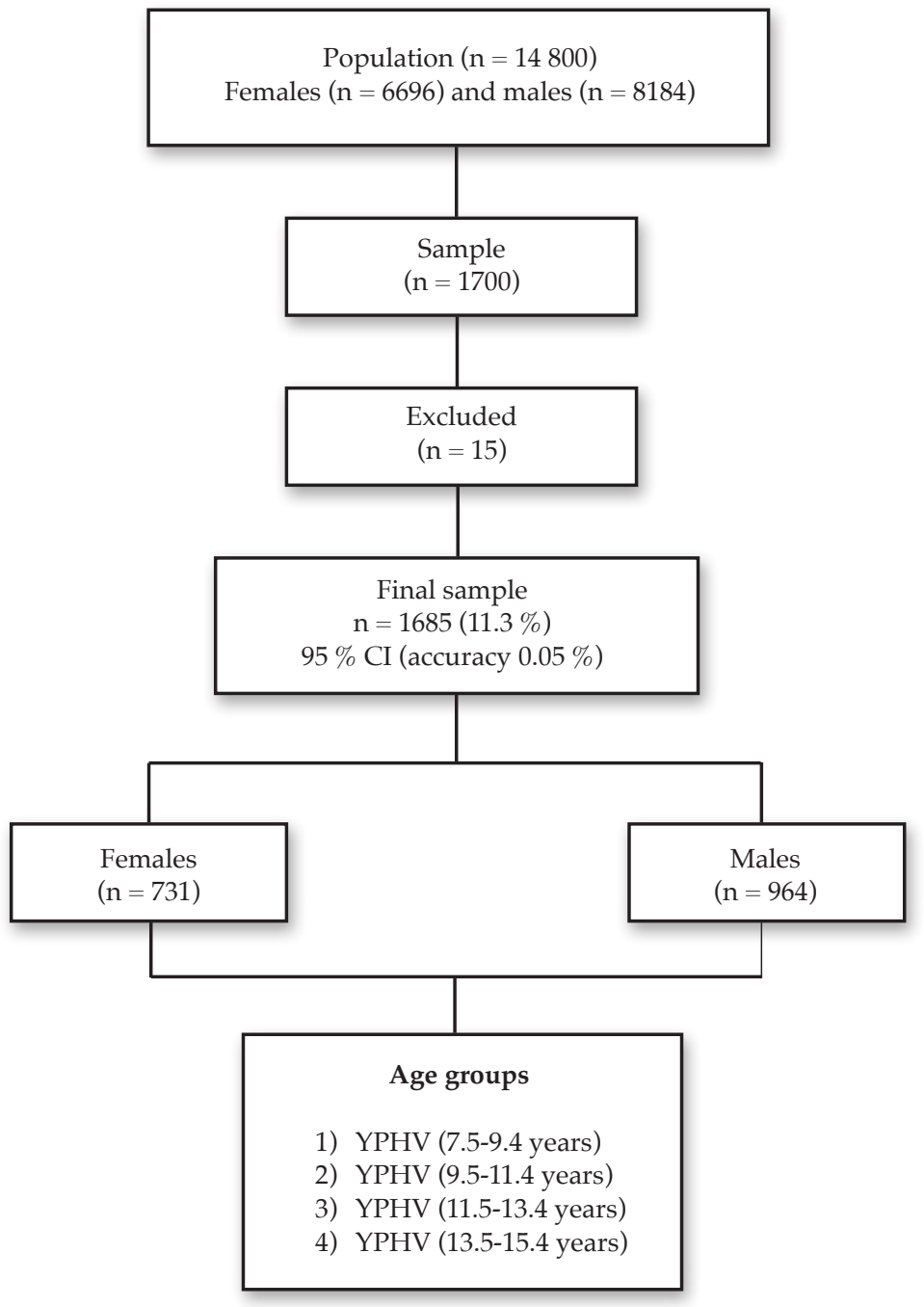

CI: confidence interval; YPHV: years from peak height velocity.

TABLE 1. Statistics (arithmetic mean and standard deviation) corresponding to anthropometric outcome measures, years from peak height velocity (maturity status), and handgrip strength by age group and sex $(n=1685)$

\begin{tabular}{|c|c|c|c|c|c|c|c|c|c|c|c|}
\hline \multirow[t]{2}{*}{ Age group } & \multirow[b]{2}{*}{$\mathbf{N}$} & \multicolumn{2}{|c|}{ Weight (kg) } & \multicolumn{2}{|c|}{ Height $(\mathrm{cm})$} & \multicolumn{2}{|c|}{ BMI $\left(\mathrm{kg} / \mathrm{m}^{2}\right)$} & \multicolumn{2}{|c|}{ YPHV (years) } & \multicolumn{2}{|c|}{ HGS (kg) } \\
\hline & & $\bar{X}$ & DE & $\bar{x}$ & DE & $\bar{X}$ & DE & $\overline{\mathrm{X}}$ & DE & $\overline{\mathrm{X}}$ & DE \\
\hline \multicolumn{12}{|l|}{ Females } \\
\hline $1(7.5-9.4)$ & 156 & 32.8 & 7.1 & 131.0 & 6.7 & 19.0 & 3.0 & $-3.2^{*}$ & 0.7 & 19.9 & 7.0 \\
\hline $2(9.5-11.4)$ & 216 & 42.1 & 9.6 & $144.1^{*}$ & 8.0 & 20.1 & 3.4 & $-1.0^{*}$ & 0.9 & 25.8 & 8.1 \\
\hline $3(11.5-13.4)$ & 191 & 52.1 & 11.5 & 154.1 & 7.2 & $21.9^{*}$ & 4.1 & $1.4^{*}$ & 1.0 & 35.2 & 14.3 \\
\hline $4(13.5-15.4)$ & 168 & 58.4 & 10.8 & $158.0^{*}$ & 6.2 & $23.3^{*}$ & 4.0 & $3.5^{*}$ & 0.9 & $46.1^{*}$ & 12.1 \\
\hline \multicolumn{12}{|l|}{ Males } \\
\hline $1(7.5-9.4)$ & 180 & 33.3 & 7.6 & 131.4 & 6.8 & 19.2 & 3.6 & -4.7 & 0.4 & 20.2 & 8.5 \\
\hline $2(9.5-11.4)$ & 213 & 41.4 & 11.6 & 140.1 & 15.9 & 20.5 & 3.9 & -3.5 & 0.7 & 26.1 & 10.4 \\
\hline $3(11.5-13.4)$ & 241 & 50.3 & 11.1 & 154.5 & 8.6 & 20.9 & 3.8 & -1.9 & 0.8 & 34.5 & 14.1 \\
\hline $4(13.5-15.4)$ & 320 & 60.1 & 12.4 & 165.1 & 8.5 & 22.0 & 3.9 & -0.3 & 0.8 & 50.7 & 18.8 \\
\hline
\end{tabular}

$\overline{\mathrm{X}}$ : average; SD: standard deviation; BMI: body mass index; *: significant difference compared to males; YPHV: years from peak height velocity (maturity status); HGS: handgrip strength. 
male and female adolescents in group 4 did not show any significant correlation after weighting the effect of maturity status. These findings are consistent because of the high prevalence of obesity observed at an early age and its reduction at an older age. In addition, the results of this study are similar to those observed in the bibliography. ${ }^{17}$

In general, studies have analyzed the association between RHGS and body composition using indirect measurements, especially BMI.

TABLE 2. Descriptive values for fat mass percentage and prevalence of obesity by age group and sex $(n=1685)$

\begin{tabular}{lccc}
\hline Age groups & \multicolumn{2}{c}{ Fat mass (\%) } & $\begin{array}{c}\text { Obesity prevalence } \\
(\%)\end{array}$ \\
\hline Females & & SD & \\
$1(7.5-9.4)$ & 35.3 & 6.1 & 93.6 \\
$2(9.5-11.4)$ & 35.0 & 6.7 & 69.0 \\
$3(11.5-13.4)$ & 33.5 & 6.9 & 49.2 \\
$4(13.5-15.4)$ & 35.6 & 6.1 & 54.2 \\
Total & 35.2 & 6.4 & 65.7 \\
Males & & & \\
$1(7.5-9.4)$ & 30.8 & 7.5 & 72.2 \\
$2(9.5-11.4)$ & 32.8 & 7.8 & 59.2 \\
$3(11.5-13.4)$ & 27.3 & 8.6 & 31.5 \\
$4(13.5-15.4)$ & 23.8 & 8.6 & 28.4 \\
Total & 26.1 & 8.7 & 44.3 \\
Total for both & 29.7 & 9.0 & 53.6 \\
\hline
\end{tabular}

$\overline{\mathbf{X}}$ : average; SD: standard deviation.

TABLE 3. Descriptive values for handgrip strength relative to body weight $(\mathrm{kg})$ for normal weight and obese children and adolescents by age and sex $(n=1685)$

\begin{tabular}{lccccccc}
\hline Age groups (years) & \multicolumn{2}{c}{ Normal weight } & \multicolumn{2}{c}{ Obese } & & Both \\
& $\overline{\mathbf{X}}$ & SD & $\overline{\mathbf{X}}$ & SD & $p$ & $\overline{\mathrm{X}}$ & SD \\
\hline Female & & & & & & & \\
$1(7.5-9.4)$ & 0.75 & 0.3 & 0.59 & 0.19 & 0.020 & 0.60 & 0.20 \\
$2(9.5-11.4)$ & 0.73 & 0.22 & 0.59 & 0.19 & $<0.001$ & 0.63 & 0.21 \\
$3(11.5-13.4)$ & 0.69 & 0.27 & 0.70 & 0.25 & 0.078 & 0.70 & 0.26 \\
$4(13.5-15.4)$ & 0.83 & 0.24 & 0.77 & 0.17 & 0.082 & 0.80 & 0.21 \\
Male & & & & & & & \\
$1(7.5-9.4)$ & 0.7 & 0.34 & 0.57 & 0.22 & 0.030 & 0.61 & 0.27 \\
$2(9.5-11.4)$ & 0.74 & 0.24 & 0.57 & 0.19 & $<0.001$ & 0.64 & 0.22 \\
$3(11.5-13.4)$ & 0.72 & 0.3 & 0.65 & 0.26 & 0.020 & 0.70 & 0.29 \\
$4(13.5-15.4)$ & 0.85 & 0.33 & 0.88 & 0.23 & 0.3996 & 0.86 & 0.31 \\
\hline
\end{tabular}

$\overline{\mathrm{X}}$ : average; SD: standard deviation.

TABLE 4. Zero-order and partial correlations by age group and sex after controlling for the effect of years from peak height velocity between fat mass percentage and relative handgrip strength $(n=1685)$

\begin{tabular}{lcccc}
\hline Age groups & \multicolumn{2}{c}{ Zero-order correlation } & \multicolumn{2}{c}{ Partial correlation (YPHV) } \\
& $\mathbf{r}$ & $\boldsymbol{p}$ & $\mathbf{r}$ & $\boldsymbol{p}$ \\
\hline Female & & & & 0.004 \\
$1(7.5-9.4)$ & -0.26 & 0.015 & -0.31 & $<0.001$ \\
$2(9.5-11.4)$ & -0.48 & $<0.001$ & -0.45 & 0.062 \\
$3(11.5-13.4)$ & -0.01 & 0.068 & 0.02 & 0.074 \\
$4(13.5-15.4)$ & -0.12 & 0.078 & -0.09 & 0.001 \\
Male & & & & $<0.001$ \\
$1(7.5-9.4)$ & -0.28 & 0.003 & -0.30 & 0.037 \\
$2(9.5-11.4)$ & -0.45 & $<0.001$ & -0.44 & 0.065 \\
$3(11.5-13.4)$ & -0.14 & 0.059 & -0.16 & -0.04 \\
$4(13.5-15.4)$ & -0.18 & 0.076 & & \\
\hline
\end{tabular}

r: Pearson correlation; YPHV: years from peak height velocity (maturity status). 
Unlike a DXA scan, BMI includes all body mass compartments (FM and fat-free mass). Probably for this reason, some studies indicate a positive association between BMI and RHGS because, in general, muscle mass is not excluded. ${ }^{17-19}$ Actually, Jürimäe et al. ${ }^{20}$ confirmed that height was the most important anthropometric outcome measure related to HGS, compared to weight and BMI, possibly because this factor showed a greater relation to fat-free mass. This research study analyzed the association after adjusting RHGS to body weight.

In addition, a recent study conducted by Cohen et al. ${ }^{21}$ showed that RHGS was inversely associated with different risk factors for cardiovascular disease in children with excess FM, but not in those with a normal FM percentage. In this regard, Ramírez-Vélez et al. ${ }^{10}$ recently demonstrated that RHGS in children and adolescents was associated with deposits of fat in the liver of children with excess FM. These results somehow support the evidence observed in our study, although in order to confirm these findings, a longitudinal study is required. In reality, in our study, children and adolescents with a higher FM had lower RHGS levels.

Therefore, most likely, regular PA levels are a determining factor in the relation between RHGS and FM. Future studies should consider the assessment of PA patterns and energy expenditure in both groups (normal weight and obesity). This information may help to analyze the results of this study more clearly.

The prevalence of obesity measured as per FM percentage (DXA) is very high (53.6\%); and according to some studies, it is above the $85^{\text {th }}$ percentile. ${ }^{16,22}$ These findings are consistent with the prevalence of overweight and obesity observed in the most recent national health survey, ${ }^{4}$ based on BMI as per the World Health Organization (WHO), which accounted for $71.2 \%$ (39.8\% for overweight and $31.2 \%$ for obesity).

RHGS for both hands was lower across all age groups and in both males and females compared to the standards published by McQuiddy et al. ${ }^{23}$ This information is similar to that reported in other studies, where RHGS was higher in older children ${ }^{18,23}$ and increased with age and, most likely, with biological maturation, especially associated with a greater increase in muscle mass in males. ${ }^{3}$ Probably for this reason, significant differences were only observed between boys and girls in age group 4, because, as of 10-11 years old, HGS increased much more among males than females. ${ }^{17,24}$ In fact, Clary et al., ${ }^{25}$ who studied developmental changes and the predictability of static strength in subjects with different maturity statuses, showed that HGS was higher among children who had achieved maturity in an early manner between 13 and 16 years old.

This study corroborated that HGS relative to body weight increases with age, except among participants between 11.5 and 13.4 years old, whose values were lower (higher among females). This age period may coincide with the onset of puberty during the biological growth and maturation process, which may actually affect body composition and strength levels in general.

Some strengths of this study worth noting are that sample selection and size are relevant and allow to generalize results to similar populations in Chile. In addition, a weakness of the study that should be noted is its cross-sectional design, which hinders the possibility of explaining causal relationships.

\section{CONCLUSION}

RHGS was confirmed to be negatively associated with FM in children and adolescents after controlling for the effect of maturity status. Future longitudinal studies are required to verify such findings.

\section{REFERENCES}

1. Lobstein T, Jackson-Leach R. Planning for the worst: estimates of obesity and comorbidities in school-age children in 2025. Pediatr Obes. 2016; 11(5):321-5.

2. World Health Organization. Final report of the Commission on Ending Childhood Obesity. Geneva: World Health Organization; 2016.

3. Malina RM, Bouchard C, Bar-Or O. Growth, Maturation and Physical Activity. $2^{\text {nd }}$ ed. Champaign: Human Kintetics; 2004.

4. Ministerio de Salud, Gobierno de Chile. Encuesta Nacional de Salud 2016-2017. Santiago: MINSAL; 2017. [Accessed on: June $\left.3^{\text {rd }}, 2020\right]$. Available at: http://epi.minsal.cl/ resultados-encuestas/.

5. Drenowatz C, Kobel S, Kettner S, Kesztyüs D, et al. Interaction of sedentary behaviour, sports participation and fitness with weight status in elementary school children. Eur J Sport Sci. 2014; 14(1):100-5.

6. Ortega FB, Ruiz JR, Castillo MJ, Sjostrom M. Physical fitness in childhood and adolescence: a powerful marker of health. Int J Obes (Lond). 2008; 32(1):1-11.

7. Mora S, Redberg RF, Cui Y, Whiteman MK, et al. Ability of exercise testing to predict cardiovascular and all-cause death in asymptomatic women: a 20-year follow-up of the lipid research clinics prevalence study. JAMA. 2003; 290(12):1600-7.

8. García-Artero E, Ortega FB, Ruiz JR, Mesa JL, et al. El perfil lipídico-metabólico en los adolescentes está más influido por la condición física que por la actividad física (estudio AVENA). Rev Esp Cardiol. 2007; 60(6):581-8.

9. Steene-Johannessen J, Anderssen SA, Kolle E, Andersen LB. Low Muscle Fitness Is Associated with Metabolic Risk in Youth. Med Sci Sports Exerc. 2009; 41(7):1361-7. 
10. Ramírez-Vélez R, Izquierdo M, Correa-Bautista JE, Tordecilla-Sanders A, et al. Grip strength moderates the association between anthropometric and body composition indicators and liver fat in youth with an excess of adiposity. J Clin Med. 2018; 7(10):347.

11. Peterson MD, Gordon PM, Smeding S, Visich P. Grip Strength Is Associated with Longitudinal Health Maintenance and Improvement in Adolescents. J Pediatr. 2018; 202:226-30.

12. Richards LG, Olson B, Palmiter-Thomas P. How Forearm Position Affects Grip Strength. Am J Occup Ther. 1996 50(2):133-8.

13. Ross WD, Marfell-Jones MJ. Kinanthropometry. In MacDougall JD, Wenger HA, Geeny HJ (eds.). Physiological testing of elite athlete. London: Human Kinetics; 1991. Pages 223-308.

14. Mirwald RL, Baxter-Jones ADG, Bailey DA, Beunen GP. An assessment of maturity from anthropometric measurements. Med Sci Sports Exerc. 2002; 34(4):689-94.

15. Cossio-Bolaños MA, Arruda M, Sulla Torres J, Urra Albornoz C, et al. Desarrollo de ecuaciones y propuesta de valores referenciales para estimar la masa grasa de niños y adolescentes chilenos. Arch Argent Pediatr. 2017; 115(5):45346.

16. Marques-Vidal P, Marcelino G, Ravasco P, Camilo ME, et al. Body fat levels in children and adolescents: Effects on the prevalence of obesity. Eur J Clin Nutr Metab. 2008; 3(6):e321-7.

17. Sartorio A, Lafortuna CL, PogliaghiS, Trecate L. The impact of gender, body dimension and body composition on handgrip strength in healthy children. J Endocrinol Invest. 2002; 25(5):431-5.
18. Zurita-Ortega F, Castro-Sánchez M, Rodríguez-Fernández $\mathrm{S}$, Cofré-Boladós $\mathrm{C}$, et al. Actividad física, obesidad y autoestima en escolares chilenos: Análisis mediante ecuaciones estructurales. Rev Med Chil. 2017; 145(3):299308.

19. Lad UP, Satyanarayana P, Shisode-Lad S, Siri CC, et al. A Study on the Correlation Between the Body Mass Index (BMI), the Body Fat Percentage, the Handgrip Strength and the Handgrip Endurance in Underweight, Normal Weight and Overweight Adolescents. J Clin Diag Res. 2013; 7(1):514.

20. Jürimäe T, Hurbo T, Jürimäe J. Relationship of handgrip strength with anthropometric and body composition variables in prepubertal children. HOMO. 2009; 60(3): 22538.

21. Cohen DD, Gómez-Arbeláez D, Camacho PA, Pinzón S, et al. Low muscle strength is associated with metabolic risk factors in Colombian children: the ACFIES study. PloS one. 2014; 9(4):e93150.

22. Laurson KR, Eisenmann JC, Welk GJ. Body Fat Percentile Curves for U.S. Children and Adolescents. Am J Prev Med. 2011; 41(4 Suppl 2):S87-92.

23. McQuiddy VA, Scheerer CR, Lavalley R, McGrath T, et al. Normative Values for Grip and Pinch Strength for 6- to 19-Year-Olds. Arch Phys Med Rehabil. 2015; 96(9):1627-33.

24. Molenaar HMT, Selles RW, Zuidam JM, Willemsen SP, et al. Growth diagrams for grip strength in children. Clin Orthop Relat Res. 2010; 468(1):217-23.

25. Taeymans J, Clarys P, Abidi H, Hebbelinck M, et al. Developmental changes and predictability of staticstrength in individuals of different maturity: A 30-year longitudinal study. J Sports Sci. 2009; 27(8):833-41. 